\title{
Estudio de caso del rendimiento académico en materias tecnológicas: la orientación de género
}

\author{
Javier Fombona Cadavieco ${ }^{1}$ \\ ORCID: http://orcid.org/0000-0001-5625-5588 \\ Fernando Aragón Gutiérrez ${ }^{1}$ \\ ORCID: https://orcid.org/0000-0003-1314-935X \\ María Ángeles Pascual Sevillano ${ }^{1}$ \\ ORCID: https://orcid.org/0000-0001-9706-2125
}

\section{Resumen}

Tradicionalmente la ciencia y tecnología se vinculaban a los estudiantes masculinos y, aunque es una división superada en determinados lugares, las investigaciones relacionadas sobre la cuestión de género siguen siendo motivo de especial interés por su trascendencia social. Este trabajo analiza las diferencias de rendimiento académico en determinadas materias en el nivel educativo de enseñanza secundaria, en un caso en España, donde una muestra de estudiantes revela un dominio interdisciplinar del rendimiento de las chicas sobre los chicos. Aunque actualmente no existen materias específicamente para hombres o mujeres, se detectan diferencias que serán significativas cuando estos alumnos aborden los estudios superiores de ingeniería. La contextualización científica ha destacado el peso del aspecto motivacional inicial en los estudiantes y los hallazgos específicos en este trabajo indican que la reducida decisión de seguir estudios superiores técnicos no correlaciona positivamente con los rendimientos académicos obtenidos en los niveles previos a la universidad. Es más, la acción educativa en enseñanzas medias reduce la diferencia en los resultados académicos de determinadas materias. A pesar de esta corriente igualitaria, las tendencias actuales se dirigen a un crecimiento limitado de las alumnas en estas especialidades formativas, y si partimos de una cantidad inicial de mujeres pequeña, es poco probable que se configuren como elemento dominante en las ingenierías.

\section{Palabras clave}

Estudios técnicos - Estudios de género - Mujeres en educación - Rendimiento académico.

1- Universidade de Oviedo, Oviedo, España. Contactos: fombona@uniovi.es; ferargu91@gmail.com; apascual@uniovi.es 


\title{
Case study of academic achievement in technological subjects: the gender orientation
}

\begin{abstract}
Traditionally science and technology have been linked to male students. Currently, this division no longer exists in many places. Research related to the gender topic still has special interest because of its social significance. This paper analyzes the differences in academic achievement in certain subjects and on the educational level of secondary schooling. Specifically we analyze a case in Spain involving a sample of students. The result reveals that girls exceed boys in mastering the academic disciplines. Although nowadays there are no subjects specific to men or women, significant differences are detected when these students deal with higher engineering studies. The scientific contextualization has highlighted the early motivation among the students, and our specific findings show the reduced decision to pursue higher technical studies, which do not correlate positively with the academic achievement obtained at pre-university levels. Moreover, the educational action in secondary schooling reduces the difference in the academic results of certain subjects. Despite this egalitarian tide, current trends turn to a limited growth of students in these training specialties, and if one starts with an initial small number of women, it is unlikely that they will become a dominant element in engineering.
\end{abstract}

\section{Keywords}

Technical studies - Gender studies - Women in education - Academic achievement.

\section{Introducción}

Los estudios sobre la cuestión de género, sobre las diferencias entre chicos y chicas y sus posibilidades en la sociedad actual, continúan estando en el núcleo de discusión en muchos contextos, bien sea por la incidencia de determinados casos en los medios (CALANDRIA, 2012), por los debates suscitados en las redes sociales (FRADE, 2010), por la controversia sobre el género de la propia semiótica de la comunicación (NAVARRO, 2011), o sencillamente, por los estereotipos que tienen nuestros jóvenes universitarios (CUBILLAS et al., 2016).

Los gobiernos están implementando políticas y destinando recursos para solventar la cuestión de la diferencia entre hombres y mujeres, por lo que parece oportuno analizar el fenómeno. Así los países escandinavos son una referencia mundial de educación igualitaria, pero difícilmente trasladable a otros contextos culturales o a otros entornos económicos menos favorecidos. Por ello, España puede considerarse un referente intermedio para distintos países en todo el mundo, dado que su economía y sociedad se 
sitúan en una posición intermedia tendente al igualitarismo, y donde su alumnado sigue referencias internacionales.

Actualmente las investigaciones exploran la superioridad de rendimiento de las chicas en materias relacionadas con la lengua y la literatura y de los chicos en matemáticas (FLECHA, 2014). Pero otros trabajos, como el realizado por la Universidad de Jaén, concluyen que las alumnas destacan por responsabilizarse más de sus fracasos y tener mejores estrategias para afrontarlos, así como para procesar la información, por lo que obtienen mejores resultados en materias relacionadas con el lenguaje, pero no encontraron diferencias de género en cuanto al rendimiento en la asignatura de Matemáticas (CEREZO; CASANOVA, 2004). Una investigación de González (2003) centrada específicamente en la asignatura de Matemáticas destaca que las chicas desempeñan tan bien o mejor que sus compañeros las tareas relacionadas con el cálculo, pero en las pruebas de aptitud y de habilidad visual y espacial muestran una diferencia en favor de los hombres. Hay estudios que marcan los rasgos diferenciales en el pensamiento icónico, enfrentado a la cultura lingüística y textual tradicional (FOMBONA, 2008), y este conocimiento de la abstracción escrita parece reafirmar esas características de género. En este sentido Echavarri, Godoy y Olaz (2007) indican que las mujeres emplean estrategias diferentes para resolver problemas complejos y aventajan a los varones en las pruebas de fluidez oral. Los varones utilizan más el razonamiento abstracto espacial, mientras que las mujeres recurren con mayor frecuencia a estrategias verbales.

En cuanto a diferencias motivacionales entre ambos sexos, el Cuestionario de Educación de Metas Académicas de Hayamizu y Weiner (1991) analiza en 2.022 adolescentes las diferencias de género en metas académicas de estudiantes de la ESO. Los resultados indican que a las chicas les motivan más las metas de logro y aprendizaje, mientras que los chicos obtienen un mayor patrón motivacional hacia metas de refuerzo social. Este planteamiento es continuado por investigadores como Delgado y otros autores (2010). En todo caso parece que la inteligencia es el mayor determinante del rendimiento académico (LYNN, 1994), pero no el único ya que otras variables de corte psicológico influyen en los resultados académicos, como los aspectos de motivación, las habilidades sociales, los rasgos de personalidad (ROBINSON, 1998), la autoeficacia percibida (BANDURA, 1986), las expectativas de logros y las variables socio-culturales (EDEL NAVARR0, 2003), los estilos cognitivos, o la propia ansiedad frente a los exámenes (MÍAS, 1999).

Varios trabajos vinculan los rendimientos globales y las formas iniciales de enfocar los estudios como elementos determinantes en el éxito escolar (VÁZQUEZ, 2009; ORTIZ; CANT0, 2013). Es una temática que parece relacionarse con los estilos de aprendizaje singulares y las estrategias cognitivas aprendidas (GUANIPA; MOGOLLÓN, 2006). Al respecto también son interesantes los análisis longitudinales realizados por Beddoes y Borrego (2011), Chou (2013), Pawley, Schimpf y Nelson (2016) que insisten en la necesidad de continuar sobre estas líneas de investigación de forma sostenida en el tiempo.

Son más limitadas las investigaciones precisas sobre los estudios técnicos y de ingeniería desde el punto de vista del género. En el último siglo estos centros tenían una mayoría de los alumnos masculinos, y aunque últimamente existe un incremento del número de chicas, esta cifra continúa siendo menor (PARDO; CALVET; MARTÍNEZ, 2016). 
Sabanes (2011) resalta el papel de las TIC en el empoderamiento de las mujeres a nivel mundial. Perales Blanco y García Guardia (2011) exploran cuestiones sobre la confluencia de la mujer y la tecnología, mostrando su deseo de avanzar hacia el equilibrio entre sexos en relación a la tecnología. Las referencias de Álvarez Lires y otros autores (2013) indican que el acceso de las mujeres a estos estudios continúa marcado por el género y tras un periodo de incremento entre las décadas finales de los 80 y el año 2000, los porcentajes de mujeres se han estancado o disminuido, y en conjunto no superan el 30\%. Existe la salvedad mundial de la ingeniería química y las tecnologías alimentarias. Sugieren que la educación técnica y científica reproduce estereotipos que refuerzan la identificación social de estudios y profesiones de este ámbito con los varones. Trabajos específicos como Borgonovi (2012) con alumnos y alumnas de 15 años sobre sus expectativas formativas en su futura edad adulta, indican que solo el 5\% de las chicas aspiran a estudiar Ingeniería o Informática mientras que, en el caso de los chicos, la cifra asciende al 18\%. Son especialmente interesantes y precisos los análisis de Llaneras (2015) donde se demuestra que las mujeres que consiguen el 59\% de los títulos universitarios en España, solamente representan el 32\% de los titulados en ingeniería.

\section{Diseño metodológico}

\section{Objetivos e hipótesis}

Actualmente género y educación son cuestiones nucleares en las políticas de la mayoría de los gobiernos, son temas que por su evolución precisan una constante revisión $y$, especialmente, la realización de investigaciones rigurosas sin predisposiciones ni sesgos. Así, el origen de esta investigación se ha centrado en la hipótesis de que los resultados de los estudiantes de secundaria correlacionan la elección de estudios técnicos superiores. Derivado del análisis de contexto surge el objetivo centrado en descubrir si existen asignaturas que pudieran asociarse a los chicos o las chicas, en los niveles de enseñanza de secundaria. Para alcanzar este objetivo, se han establecido dos sub-objetivos:
a) Conocer cuáles son las materias de estudio donde los chicos y las chicas, tienen un mejor rendimiento académico.
b) Averiguar si la acción educativa, esto es, el hecho de ser estudiante durante un tiempo, agudiza o atenúa estas diferencias de rendimiento.
c) Verificar el tipo de variaciones en las materias tecnológicas.
d) Comparar los resultados con la literatura científica relacionada existente.

\section{Método}

Este trabajo se realiza dentro de una amplia actividad investigadora de la Facultad de Formación del Profesorado y Educación de la Universidad de Oviedo (España) donde se abordan las claves e influencia del sistema educativo en la sociedad contemporánea, específicamente en Europa y en el proceso de educación denominado a lo largo de la vida, eje clave en las políticas continentales actuales. La tarea se centra en recopilar datos 
rigurosos sobre temas que habitualmente son objeto de polémica social, en este caso se analiza la diferencia entre sexos desde una perspectiva descriptiva. Se considera un trabajo con carácter explicativo, ya que pretende averiguar las implicaciones del hecho diferencial de género descrito, a partir de unos datos precisos derivados de los resultados académicos. Para ello se analiza el caso de las asignaturas en un caso de un centro público donde se imparten estudios en los niveles de enseñanza secundaria española. El universo de la presente investigación lo conforman todos los estudiantes de enseñanzas medias en España. La población objetivo de investigación se centra en el análisis de 63 estudiantes $2^{\circ}$ curso de secundaria, con edades comprendidas entre los 13, 14 y 15 años. Es una muestra aleatoria de estudiantes de un Instituto de Educación Secundaria en la región de Asturias, al norte de España, de los que se ha tenido acceso a los documentos privados que contienen sus resultados académicos, en el curso 2015-2016. Se trata de tres grupos de alumnos, que atendiendo al género se distribuyen como se indica en la Tabla 1.

Tabla 1- Distribución de la muestra

\begin{tabular}{c|ccc:cc}
\hline & Total estudiantes & N $^{0}$ alumnas & \multicolumn{1}{c}{ N $^{\mathbf{a}}$ alumnos } \\
\hline Grupo A & 21 & $\vdots$ & 12 & 9 \\
\hline Grupo B & 20 & & 8 & 12 \\
\hline Grupo C & 22 & $\vdots$ & 9 & 13 \\
\hline Total & 63 & $\vdots$ & 29 & 34 \\
\hline Estudiantes absentistas & 9 & $\vdots$ & 2 & 7 \\
\hline
\end{tabular}

Fuente: Elaboración propia.

Se observa que el número total de chicas y de chicos es similar, con un total de 29 alumnas y 34 alumnos, lo que proporciona muestras equivalentes, aunque reducidas. El estudio se realizará con los datos resultantes de la evaluación del rendimiento académico y calificaciones obtenidas en las diferentes asignaturas. Con relación a las características sobre fiabilidad y validez del estudio, se debe indicar que los datos han sido extraídos de los expedientes personales y que estos documentos reflejan unos resultados obtenidos con procedimientos rigurosos de cuantificación de rendimiento académico, tales como una evaluación colegiada por el grupo de docentes que analiza estas calificaciones. Por otro lado, toda esta información es tratada con elevados niveles de seguridad, precisión y confidencialidad ya que de ella deriva el futuro profesional de cada estudiante.

Así mismo se ha considerado el problema del absentismo y abandono escolar que podría condicionar las estadísticas de los resultados obtenidos. Por ello en las comparativas estadísticas no se han analizado los casos de estudiantes que habitualmente, y por causas diversas ajenas a la coyuntura académica, no asisten al aula, excluyéndolos en el tratamiento de datos. En esta línea se han considerado personas absentistas aquellas cuya calificación media entre todas las asignaturas sea menor de un dos. Obsérvese que la escala de evaluación española oscila entre 0 puntos como resultado mínimo, y un máximo de 10 puntos, siendo habitual el mínimo suficiente de 5 puntos para el progreso y promoción entre nivel y curso. Una vez excluidas 2 alumnas y 7 alumnos dentro de esta categoría, se concluye la selección en 63 casos, con un número de 29 son chicas y 34 son chicos. 
Para comparar los resultados con la literatura científica relacionada existente se ha trabajado sobre el repositorio de investigación científica Web of Science, WoS, indexado por Thomson, que cataloga referencias científicas de alto impacto con más de 12.000 artículos agrupados en ciencias, ciencias sociales, artes y humanidades. Esta parte tiene un carácter cualitativo y se ha realizado un contraste simultáneo entre concordancias internas en el equipo de la Universidad de Oviedo, actuando como dobles expertos, y según la literatura sobre confiabilidad entre evaluadores se ha seguido el índice kappa de Cohen. Este coeficiente considera los contenidos relevantes no sólo por acuerdos debidos a la casualidad, por ello se cuantificó sólidamente la concordancia entre esos examinadores y sus categorías cualitativas. El análisis de las referencias se ha centrado en los descriptores de temática education y gender. Como este análisis cuantitativo generaba demasiados registros (superaba los 63.000) se optó por una elección muestral bajo criterios de temporalidad y conveniencia, este planteamiento es normalmente aceptado cuando nos enfrentamos ante la abundancia de información de estos casos (ÁVILA, 1999). Se hizo una búsqueda más precisa a partir del año 2016 excluyendo los documentos de las bases orientadas a la medicina (Medline), y a la tecnología (Science Technology). Esto permitió un análisis de 53 documentos relacionados. Se utilizaron dos instrumentos, la herramienta Atlas.ti-7.5.12 y la propia codificación de la base WoS que permitía el control de resultados manejando, entre otros, los operadores de búsqueda $y$ (and) para registros con todos los términos; o (or) para localizar registros con algún término.

\section{Técnicas e instrumentos de investigación de los resultados académicos}

La investigación se basa en el análisis de datos académicos constatados, esto es, las calificaciones oficiales de los estudiantes que forman parte de la muestra. En cada estudiante se cuantifican sus resultados académicos con el fin de alcanzar los objetivos, atendiendo a las siguientes variables:

- Diferencia entre la calificación media total y la nota media de una materia objeto de estudio. Este indicador marca la diferencia entre resultados de chicos y de chicas en una determinada asignatura, comparada con la diferencia entre la nota media total de chicos y chicas. Esta medición nos ayuda a considerar si existen materias específicas de chicos donde estos estudiantes obtienen mejores resultados o es que esos estudiantes obtienen mejores resultados en todas las asignaturas. Así comparamos los resultados en cada asignatura y las variaciones con el resto de materias.

- Alumnos y alumnas con mejores resultados. Mediante este indicador se observa cuál es el género de los estudiantes con mejores calificaciones en una determinada asignatura.

- Alumnos y alumnas con peores resultados. Mediante este indicador se observará cuál es el género de los estudiantes con peores resultados en una determinada asignatura.

- Niveles de evolución en los resultados académicos para cada uno de los participantes. Esto nos permite observar el sentido del cambio y la cantidad de mejora en el rendimiento académico en un mismo grupo diferenciado por materias. 
Se han analizado los resultados del alumnado de tres secciones de segundo curso de enseñanza secundaria. Para guardar su anonimato se sitúan siguiendo con un código formado por una letra, $\mathrm{M}$ o F, según se trate de una alumna o de un alumno respectivamente, seguido de su número en clase. Así, el M7A, se refiere a un chico (M), el número 7 de su clase, en el grupo A. Se ha analizado también la evolución de cada estudiante, diferenciando los resultados obtenidos en cada caso tras la primera mitad del curso y luego tras la segunda mitad, es decir, las calificaciones de la primera evaluación y de la evaluación final. Se realiza el tratamiento estadístico de resultados con el programa SPSS v. 22.0.

\section{Resultados académicos}

En tabla 2 aparecen reflejados los resultados académicos obtenidos en la primera evaluación por el total de estudiantes, 63, y diferenciados por materias. En la columna de la derecha se observa la media estadística en cada caso.

Tabla 2- Resultados de la primera evaluación (escala 0 a 10 puntos)

\begin{tabular}{|c|c|c|c|c|c|c|c|c|c|}
\hline Chicos & Naturales & Sociales & E. F. & Lengua & Matemáticas & Música & Tecnología & Inglés & Media \\
\hline M1A & 1 & 1 & 2 & 1 & 1 & 3 & 3 & 3 & 1,875 \\
\hline $\mathrm{M} 2 \mathrm{~A}$ & 6 & 7 & 7 & 6 & 4 & 8 & 5 & 7 & 6,25 \\
\hline M3A & 1 & 1 & 1 & 1 & 1 & 1 & 1 & 1 & 1 \\
\hline M4A & 1 & 1 & 1 & 2 & 1 & 3 & 1 & 5 & 1,875 \\
\hline M5A & 4 & 7 & 7 & 3 & 1 & 7 & 4 & 3 & 4,5 \\
\hline M6A & 3 & 5 & 7 & 3 & 4 & 6 & 6 & 4 & 4,75 \\
\hline M15A & 5 & 5 & 6 & 4 & 1 & 6 & 4 & 4 & 4,375 \\
\hline M19A & 7 & 8 & 8 & 6 & 5 & 6 & 7 & 6 & 6,625 \\
\hline $\mathrm{M} 1 \mathrm{~B}$ & 1 & 1 & 1 & 0 & 1 & 2 & 1 & 1 & 1 \\
\hline $\mathrm{M} 2 \mathrm{~B}$ & 5 & 7 & 5 & 4 & 4 & 8 & 6 & 6 & 5,625 \\
\hline M3B & 5 & 6 & 7 & 5 & 4 & 8 & 6 & 6 & 5,875 \\
\hline M4B & 5 & 6 & 5 & 4 & 4 & 8 & 7 & 4 & 5,375 \\
\hline M5B & 3 & 6 & 8 & 3 & 3 & 6 & 5 & 2 & 4,5 \\
\hline M8B & 6 & 7 & 9 & 6 & 5 & 8 & 6 & 6 & 6,625 \\
\hline M10B & 6 & 6 & 6 & 6 & 5 & 6 & 6 & 6 & 5,875 \\
\hline M14B & 1 & 1 & 1 & 1 & 1 & 1 & 1 & 1 & 1 \\
\hline M15B & 5 & 5 & 7 & 6 & 5 & 8 & 6 & 6 & 6 \\
\hline M17B & 4 & 5 & 6 & 3 & 2 & 6 & 6 & 4 & 4,5 \\
\hline M19B & 2 & 4 & 6 & 1 & 3 & 6 & 2 & 5 & 3,625 \\
\hline M20B & 6 & 7 & 8 & 5 & 6 & 8 & 8 & 8 & 7 \\
\hline $\mathrm{M} 21 \mathrm{~B}$ & 1 & 1 & 1 & 1 & 1 & 6 & 1 & 1 & 1,625 \\
\hline M2C & 5 & 3 & 3 & 4 & 3 & 6 & 4 & 7 & 4,375 \\
\hline M4C & 6 & 5 & 6 & 5 & 4 & 8 & 6 & 6 & 5,75 \\
\hline M5C & 4 & 5 & 5 & 3 & 2 & 6 & 5 & 5 & 4,375 \\
\hline M6C & 6 & 7 & 7 & 5 & 4 & 9 & 5 & 7 & 6,25 \\
\hline
\end{tabular}


Javier Fombona Cadavieco; Fernando Aragón Gutiérrez; María Ángeles Pascual Sevillano

\begin{tabular}{|c|c|c|c|c|c|c|c|c|c|}
\hline $\mathrm{M} 9 \mathrm{C}$ & 9 & 8 & 8 & 7 & 7 & 7 & 8 & 9 & 7,875 \\
\hline M11C & 6 & 5 & 7 & 5 & 4 & 8 & 6 & 8 & 6,125 \\
\hline $\mathrm{M} 12 \mathrm{C}$ & 6 & 6 & 9 & 6 & 7 & 8 & 7 & 8 & 7,125 \\
\hline M15C & 1 & 1 & 1 & 1 & 1 & 2 & 1 & 1 & 1,125 \\
\hline M16C & 1 & 1 & 4 & 1 & 1 & 6 & 3 & 5 & 2,75 \\
\hline M18C & 7 & 6 & 7 & 5 & 5 & 6 & 7 & 6 & 6,125 \\
\hline M19C & 3 & 5 & 5 & 1 & 1 & 5 & 4 & 4 & 3,5 \\
\hline M21C & 8 & 8 & 9 & 6 & 5 & 8 & 8 & 8 & 7,5 \\
\hline $\mathrm{M} 22 \mathrm{C}$ & 8 & 8 & 9 & 6 & 8 & 10 & 8 & 9 & 8,25 \\
\hline Chicas & Naturales & Sociales & E. F. & Lengua & Matemáticas & Música & Tecnología & Inglés & Media \\
\hline F7A & 9 & 7 & 9 & 6 & 6 & 10 & 8 & 9 & 8 \\
\hline F8A & 6 & 5 & 6 & 4 & 5 & 10 & 5 & 9 & 6,25 \\
\hline F9A & 6 & 8 & 9 & 5 & 6 & 10 & 7 & 7 & 7,25 \\
\hline F10A & 7 & 7 & 8 & 6 & 3 & 10 & 7 & 8 & 7 \\
\hline $\mathrm{F} 11 \mathrm{~A}$ & 4 & 4 & 6 & 4 & 4 & 5 & 5 & 6 & 4,75 \\
\hline F12A & 5 & 7 & 8 & 5 & 4 & 10 & 9 & 4 & 6,5 \\
\hline F13A & 5 & 7 & 7 & 5 & 7 & 9 & 8 & 7 & 6,875 \\
\hline F14A & 6 & 6 & 8 & 5 & 5 & 8 & 7 & 5 & 6,25 \\
\hline F16A & 7 & 7 & 9 & 5 & 4 & 10 & 9 & 6 & 7,125 \\
\hline F17A & 9 & 10 & 10 & 8 & 8 & 10 & 9 & 10 & 9,25 \\
\hline F18A & 6 & 7 & 8 & 6 & 5 & 9 & 7 & 7 & 6,875 \\
\hline F20A & 7 & 7 & 8 & 4 & 3 & 10 & 7 & 5 & 6,375 \\
\hline F6B & 10 & 9 & 9 & 8 & 7 & 10 & 8 & 8 & 8,625 \\
\hline F7B & 3 & 4 & 8 & 5 & 5 & 8 & 4 & 7 & 5,5 \\
\hline $\mathrm{F9B}$ & 10 & 10 & 10 & 9 & 9 & 10 & 9 & 9 & 9,5 \\
\hline $\mathrm{F} 11 \mathrm{~B}$ & 4 & 5 & 5 & 6 & 2 & 8 & 5 & 9 & 5,5 \\
\hline F12B & 7 & 7 & 8 & 7 & 6 & 9 & 8 & 9 & 7,625 \\
\hline F13B & 2 & 4 & 2 & 3 & 2 & 6 & 1 & 3 & 2,875 \\
\hline F16B & 10 & 9 & 9 & 8 & 8 & 10 & 9 & 9 & 9 \\
\hline F18B & 10 & 9 & 9 & 8 & 8 & 10 & 9 & 10 & 9,125 \\
\hline $\mathrm{F} 1 \mathrm{C}$ & 1 & 1 & 1 & 1 & 1 & 2 & 1 & 1 & 1,125 \\
\hline F3C & 7 & 8 & 9 & 7 & 4 & 9 & 7 & 7 & 7,25 \\
\hline F7C & 9 & 9 & 9 & 7 & 7 & 10 & 8 & 10 & 8,625 \\
\hline F8C & 1 & 1 & 3 & 1 & 1 & 1 & 1 & 1 & 1,25 \\
\hline F10C & 8 & 7 & 9 & 6 & 6 & 9 & 8 & 8 & 7,625 \\
\hline F13C & 10 & 9 & 10 & 8 & 8 & 10 & 8 & 9 & 9 \\
\hline F14C & 4 & 7 & 5 & 4 & 3 & 9 & 6 & 5 & 5,375 \\
\hline F17C & 4 & 5 & 2 & 1 & 3 & 5 & 1 & 3 & 3 \\
\hline F20C & 10 & 9 & 10 & 8 & 7 & 10 & 9 & 9 & 9 \\
\hline
\end{tabular}

E.F.: Educación Física.

Fuente: Elaboración propia. 
Por otro lado, en Tabla 3 aparecen reflejados los resultados académicos obtenidos en la segunda evaluación por esos mismos 63 estudiantes, también diferenciados por materias. En la columna de la derecha se observa la media estadística en cada caso.

Tabla 3- Resultados de la evaluación final (escala 0 a 10 puntos)

\begin{tabular}{|c|c|c|c|c|c|c|c|c|c|}
\hline Chicos & Naturales & Sociales & E. F. & Lengua & Matemáticas & Música & Tecnología & Inglés & Media \\
\hline M1A & 1 & 1 & 1 & 2 & 1 & 5 & 1 & 1 & 1,625 \\
\hline M2A & 8 & 6 & 6 & 5 & 5 & 6 & 7 & 8 & 6,375 \\
\hline M3A & 1 & 1 & 1 & 1 & 1 & 1 & 1 & 1 & 1 \\
\hline M4A & 1 & 1 & 1 & 1 & 3 & 4 & 1 & 2 & 1,75 \\
\hline M5A & 7 & 5 & 8 & 4 & 1 & 5 & 5 & 5 & 5 \\
\hline M6A & 7 & 5 & 7 & 4 & 2 & 6 & 5 & 3 & 4,875 \\
\hline M15A & 5 & 3 & 7 & 3 & 1 & 5 & 3 & 3 & 3,75 \\
\hline M19A & 6 & 7 & 7 & 6 & 6 & 9 & 6 & 6 & 6,625 \\
\hline M1B & 1 & 1 & 1 & 1 & 1 & 2 & 1 & 0 & 1 \\
\hline $\mathrm{M} 2 \mathrm{~B}$ & 4 & 5 & 7 & 4 & 4 & 5 & 6 & 4 & 4,875 \\
\hline M3B & 7 & 7 & 7 & 5 & 3 & 6 & 6 & 3 & 5,5 \\
\hline M4B & 6 & 6 & 6 & 4 & 5 & 6 & 6 & 4 & 5,375 \\
\hline M5B & 5 & 5 & 6 & 3 & 2 & 5 & 5 & 2 & 4,125 \\
\hline M8B & 6 & 6 & 8 & 7 & 4 & 8 & 7 & 6 & 6,5 \\
\hline $\mathrm{M} 10 \mathrm{~B}$ & 6 & 6 & 6 & 6 & 4 & 5 & 7 & 4 & 5,5 \\
\hline M14B & 1 & 1 & 1 & 1 & 1 & 1 & 1 & 1 & 1 \\
\hline M15B & 5 & 5 & 7 & 4 & 4 & 6 & 5 & 5 & 5,125 \\
\hline M17B & 5 & 4 & 6 & 2 & 2 & 5 & 7 & 5 & 4,5 \\
\hline M19B & 2 & 2 & 4 & 1 & 2 & 4 & 2 & 3 & 2,5 \\
\hline M20B & 6 & 6 & 7 & 5 & 6 & 8 & 8 & 8 & 6,75 \\
\hline $\mathrm{M} 21 \mathrm{~B}$ & 1 & 1 & 1 & 1 & 1 & 5 & 1 & 1 & 1,5 \\
\hline M2C & 5 & 5 & 5 & 5 & 2 & 5 & 6 & 4 & 4,625 \\
\hline M4C & 5 & 5 & 6 & 3 & 4 & 7 & 6 & 7 & 5,375 \\
\hline M5C & 3 & 5 & 5 & 2 & 2 & 5 & 6 & 5 & 4,125 \\
\hline M6C & 5 & 6 & 6 & 5 & 4 & 8 & 6 & 7 & 5,875 \\
\hline M9C & 9 & 9 & 6 & 8 & 7 & 7 & 8 & 8 & 7,75 \\
\hline M11C & 7 & 7 & 6 & 5 & 7 & 7 & 6 & 8 & 6,625 \\
\hline $\mathrm{M} 12 \mathrm{C}$ & 7 & 7 & 8 & 5 & 6 & 7 & 7 & 8 & 6,875 \\
\hline M15C & 1 & 1 & 1 & 1 & 1 & 1 & 1 & 1 & 1 \\
\hline M16C & 2 & 1 & 7 & 1 & 1 & 5 & 3 & 4 & 3 \\
\hline M18C & 6 & 6 & 6 & 5 & 7 & 7 & 6 & 7 & 6,25 \\
\hline M19C & 3 & 5 & 5 & 2 & 1 & 5 & 4 & 2 & 3,375 \\
\hline
\end{tabular}




\begin{tabular}{|c|c|c|c|c|c|c|c|c|c|}
\hline M21C & 7 & 8 & 8 & 5 & 4 & 9 & 7 & 8 & 7 \\
\hline M22C & 8 & 9 & 8 & 9 & 7 & 9 & 7 & 9 & 8,25 \\
\hline Chicas & Naturales & Sociales & E. F. & Lengua & Matemáticas & Música & Tecnología & Inglés & Media \\
\hline F7A & 7 & 8 & 7 & 6 & 6 & 9 & 8 & 8 & 7,375 \\
\hline F8A & 6 & 5 & 7 & 5 & 5 & 8 & 6 & 5 & 5,875 \\
\hline F9A & 6 & 7 & 8 & 5 & 5 & 8 & 8 & 6 & 6,625 \\
\hline F10A & 6 & 7 & 7 & 7 & 3 & 8 & 6 & 8 & 6,5 \\
\hline $\mathrm{F} 11 \mathrm{~A}$ & 2 & 2 & 5 & 5 & 4 & 3 & 3 & 4 & 3,5 \\
\hline F12A & 6 & 8 & 7 & 5 & 4 & 7 & 6 & 5 & 6 \\
\hline F13A & 7 & 8 & 8 & 8 & 6 & 7 & 7 & 7 & 7,25 \\
\hline $\mathrm{F} 14 \mathrm{~A}$ & 5 & 6 & 8 & 5 & 5 & 8 & 6 & 4 & 5,875 \\
\hline F16A & 8 & 8 & 8 & 5 & 6 & 9 & 9 & 6 & 7,375 \\
\hline F17A & 9 & 10 & 9 & 9 & 8 & 10 & 10 & 10 & 9,375 \\
\hline F18A & 6 & 6 & 8 & 5 & 5 & 8 & 6 & 6 & 6,25 \\
\hline F20A & 8 & 8 & 7 & 5 & 3 & 6 & 5 & 5 & 5,875 \\
\hline F6B & 9 & 10 & 7 & 9 & 8 & 10 & 9 & 9 & 8,875 \\
\hline F7B & 5 & 6 & 7 & 6 & 4 & 5 & 5 & 6 & 5,5 \\
\hline F9B & 9 & 10 & 9 & 10 & 9 & 10 & 9 & 9 & 9,375 \\
\hline $\mathrm{F} 11 \mathrm{~B}$ & 5 & 3 & 6 & 7 & 2 & 7 & 5 & 8 & 5,375 \\
\hline $\mathrm{F} 12 \mathrm{~B}$ & 6 & 8 & 8 & 6 & 5 & 8 & 8 & 8 & 7,125 \\
\hline F13B & 2 & 1 & 2 & 2 & 1 & 3 & 1 & 2 & 1,75 \\
\hline F16B & 8 & 9 & 9 & 8 & 8 & 10 & 9 & 10 & 8,875 \\
\hline F18B & 8 & 10 & 8 & 9 & 9 & 10 & 10 & 10 & 9,25 \\
\hline F1C & 1 & 1 & 1 & 1 & 1 & 2 & 1 & 1 & 1,125 \\
\hline F3C & 9 & 9 & 8 & 7 & 6 & 9 & 7 & 8 & 7,875 \\
\hline F7C & 9 & 10 & 8 & 9 & 7 & 8 & 8 & 9 & 8,5 \\
\hline F8C & 1 & 1 & 1 & 1 & 1 & 1 & 1 & 1 & 1 \\
\hline F10C & 7 & 9 & 8 & 6 & 6 & 6 & 8 & 8 & 7,25 \\
\hline F13C & 10 & 9 & 9 & 9 & 9 & 10 & 9 & 9 & 9,25 \\
\hline F14C & 5 & 6 & 6 & 2 & 3 & 6 & 5 & 5 & 4,75 \\
\hline F17C & 4 & 5 & 5 & 3 & 2 & 3 & 1 & 1 & 3 \\
\hline F20C & 9 & 9 & 9 & 8 & 8 & 10 & 8 & 10 & 8,875 \\
\hline
\end{tabular}

E.F.: Educación Física.

Fuente: Elaboración propia.

\section{Resultados en la primera evaluación}

Atendiendo a la diferencia entre los datos medios de chicas y chicos en las diferentes asignaturas, se obtienen los siguientes resultados indicados en Tabla 4. Destaca que las 
alumnas obtienen mejores resultados en todas las asignaturas, como mínimo 1,06 puntos más y como máximo 1,96 puntos más. Siendo, en este caso Música la asignatura en la que hay una mayor diferencia y Educación Física en la que la diferencia es menor.

Tabla 4- Notas medias (escala 0 a 10 puntos) por asignatura y género

\begin{tabular}{c|c|c:c}
\hline $1^{\text {a } \text { evaluación }}$ & Media chicas & Media chicos & Diferencia \\
\hline Naturales & 6,85 & 5,08 & 1,77 \\
\hline Sociales & 7,15 & 5,72 & 1,43 \\
\hline Educación Física & 7,78 & 6,72 & 1,06 \\
\hline Lengua & 5,85 & 4,32 & 1,53 \\
\hline Matemáticas & 5,37 & 3,84 & 1,53 \\
\hline Música & 9,04 & 7,08 & 1,96 \\
\hline Tecnología & 6,96 & 5,64 & 1,32 \\
\hline Inglés & 7,33 & 5,76 & 1,57 \\
\hline
\end{tabular}

Fuente: Elaboración propia.

Se han analizado los casos de estudiantes -alumnos y alumnas- con mejores y peores resultados en las diferentes asignaturas. Se obtienen los siguientes datos reflejados en tabla 5.

Tabla 5- Calificaciones de cada asignatura por género y materia $1^{\mathrm{a}}$ evaluación

\begin{tabular}{|c|c|c|c|}
\hline $1^{\mathrm{a}}$ evaluación & Altas calificaciones & Chicas & Chicos \\
\hline Naturales & F6B, F9B, F16B, F18B, F13C, F20C. & 6 & 0 \\
\hline Sociales & F17A, F6B, F9B, F16B, F18B, F7C, F13C, F20C. & 8 & 0 \\
\hline Educación Física & Más de 10 & 13 & 3 \\
\hline Lengua & F17A, F6B, F9B, F16B, F18B, F13C, F20C & 7 & 0 \\
\hline Matemáticas & F17A, F9B, F16B, F18B, F13C, M22C & 5 & 1 \\
\hline Música & Más de 10 & 15 & 1 \\
\hline Tecnología & F12A, F16A, F17A, F9B, F1622B, F18B, F20C. & 7 & 0 \\
\hline Inglés & Más de 10 & 12 & 1 \\
\hline $1^{a}$ evaluación & Bajas calificaciones & Chicas & Chicos \\
\hline Naturales & M6A, M5B, F7B, F13B, M19B, M16C, M19C. & 2 & 5 \\
\hline Sociales & F11A, F7B, F13B, M19B, M2C, M16C. & 3 & 3 \\
\hline Educación Física & M2B, M4B, F11B, F13B, M2C, F14C, M16C, F17C, M19C. & 4 & 5 \\
\hline Lengua & Más de 10 & 3 & 7 \\
\hline Matemáticas & M5A, M15A, F11B, F13B, M17B, M5C, M16C, M19C, & 2 & 6 \\
\hline Música & Más de 10 & 3 & 12 \\
\hline Tecnología & M5A, M15A, F7B, F13B, M19B, M2C, M16C, F17C, M19C, & 3 & 6 \\
\hline Inglés & Más de 10 & 3 & 7 \\
\hline
\end{tabular}

Fuente: Elaboración propia. 


\section{Resultados en la evaluación final}

Atendiendo al primer factor, es decir a la diferencia entre los resultados de todos los chicos y chicas en las diferentes asignaturas, se obtienen los datos reflejados en siguiente Tabla 6 .

Tabla 6- Calificaciones medias (escala 0 a 10 puntos) por asignatura y sexo en la evaluación final

\begin{tabular}{|c|c|c|c|}
\hline Evaluación final & Media chicas & Media chicos & Diferencia \\
\hline Naturales & 6,70 & 5,56 & 1,14 \\
\hline Sociales & 7,30 & 5,48 & 1,82 \\
\hline Educación Física & 7,33 & 6,48 & 0,85 \\
\hline Lengua & 6,33 & 4,24 & 2,09 \\
\hline Matemáticas & 5,44 & 3,68 & 1,76 \\
\hline Música & 7,63 & 6,32 & 1,31 \\
\hline Tecnología & 6,74 & 5,72 & 1,02 \\
\hline Inglés & 6,89 & 5,36 & 1,53 \\
\hline
\end{tabular}

Fuente: Elaboración propia.

Se observa cómo se mantienen los mejores resultados en las alumnas en todas las materias, en este caso, como mínimo 0,85 puntos más en Educación Física y como máximo 2,09 puntos en Lengua.

Atendiendo al siguiente criterio, se buscan los estudiantes con mejores y peores resultados en las diferentes asignaturas. Se obtienen los siguientes datos de Tabla 7.

Tabla 7- Calificaciones de cada asignatura (escala 0 a 10 puntos) según género y materia en evaluación final

\begin{tabular}{|c|c|c|c|}
\hline Evaluación final & Mejores resultados & Chicas & Chicos \\
\hline Naturales & F17A, F6B, F9B, F3C, F7C, M9C, F13C, F20C. & 7 & 1 \\
\hline Sociales & F17A, F6B, F9B, F18B, F7C & 5 & 0 \\
\hline Educación Física & F17A, F9B, F16B, F13C, F20C. & 5 & 0 \\
\hline Lengua & F17A, F6B, F9B, F18B, F7C, F13C & 6 & 0 \\
\hline Matemáticas & F17A, F6B, F9B, F16B, F18B, F13C, F20C & 7 & 0 \\
\hline Música & F17A, F6B, F9B, F16B, F18B, F13C & 6 & 0 \\
\hline Tecnología & F16A, F17A, F6B, F9B, F16B, F18B, F20C. & 7 & 0 \\
\hline Inglés & Más de 10 & 9 & 1 \\
\hline Evaluación final & Peores resultados & Chicas & Chicos \\
\hline Naturales & F11A, M19B, M5C, M16C, M19C. & 1 & 4 \\
\hline Sociales & F11A, M15A, F11B, M19B, M16C & 3 & 2 \\
\hline Educación Física & F11A, M19B, M2C, M5C, F17C, M19C. & 2 & 4 \\
\hline Lengua & M15A, M5B, M17B, M19B, M4C, M5C, F14C, M16C, F17C, M19C. & 2 & 8 \\
\hline Matemáticas & M5A, M15A, M19B, M16C, M19C & 0 & 5 \\
\hline Música & Más de 10 & 3 & 11 \\
\hline Tecnología & F11A, M15A, M19B, M16C, F17C. & 2 & 3 \\
\hline Inglés & M6A, M15A, M3B, M5B, M19B, F17C, M19C & 3 & 7 \\
\hline
\end{tabular}

Fuente: Elaboración propia. 
Es interesante observar el progreso obtenido en cada materia, según refleja la tabla 4 , en donde se observan ligeras reducciones en materias como Tecnología e incrementos en el caso de Lengua.

Tabla 8- Resultados entre evaluaciones

\begin{tabular}{|c|c|c|c|c|c|c|c|c|c|}
\hline $1^{\mathrm{a}}$ evaluación & Naturales & Sociales & E. F. & Lengua & Matem. & Música & Tecnol. & Inglés & Media \\
\hline Media M & 5,08 & 5,72 & 6,72 & 4,32 & 3,84 & 7,08 & 5,64 & 5,76 & 5,52 \\
\hline Media F & 6,85 & 7,15 & 7,78 & 5,85 & 5,37 & 9,04 & 6,96 & 7,33 & 7,04 \\
\hline Diferencia & 1,77 & 1,43 & 1,06 & 1,53 & 1,53 & 1,96 & 1,32 & 1,57 & 1,52 \\
\hline Evaluación final & Naturales & Sociales & E. F. & Lengua & Matem. & Música & Tecnol. & Inglés & Media \\
\hline Media M & 5,56 & 5,48 & 6,48 & 4,24 & 3,68 & 6,32 & 5,72 & 5,36 & 5,355 \\
\hline Media F & 6,70 & 7,30 & 7,33 & 6,33 & 5,44 & 7,63 & 6,74 & 6,89 & 6,80 \\
\hline Diferencia & 1,14 & 1,82 & 0,85 & 2,09 & 1,76 & 1,31 & 1,02 & 1,53 & 1,44 \\
\hline $\begin{array}{l}\text { Variación entre } 1^{\text {a }} \text { y } \\
\text { evaluación final }\end{array}$ & $-0,63$ & $+0,39$ & $-0,21$ & $+0,56$ & $+0,23$ & $-0,65$ & $-0,30$ & -0.04 & \\
\hline
\end{tabular}

E.F.: Educación Física.

Fuente: Elaboración propia.

Se observa que en la primera evaluación los resultados académicos generales medios son superiores en las chicas que en los chicos 1,5 puntos. Esta diferencia sigue constante en la evaluación final.

Por otro lado, esa supremacía se mantiene también en la asignatura de Tecnología aunque con una diferencia dos décimas menos: 1,3 puntos en la evaluación primera. Pero con el paso del tiempo educativo en la evaluación final de la asignatura de Tecnología la diferencia se reduce otras 3 décimas, quedando en 1,0 punto.

El estudio de la evolución de resultados académicos en el tiempo denota una tendencia a reducir las diferencias académicas y de género a medida en que avanza el curso. Estos datos son especialmente significativos ya que se refieren a una muestra de grupos equivalentes cuantificados tras el paso de tan sólo 3 meses y se ha producido una reducción de 0,5 puntos en el total de medias de resultados.

\section{Resultados en la búsqueda de literatura científica relacionada}

La búsqueda de literatura científica relacionada ha podido detectar una producción sostenida en el tiempo en la última década y dado que ha sido una terea extensa en la cantidad de registros, ha sido necesaria su limitación al periodo 2016-2017, donde destacamos los siguientes tópicos relacionados.

- Necesidad de estudios específicos, parcializados y centrados en casos. En primer lugar, es necesario analizar por separado las múltiples variables del fenómeno género y educación, ya que se pueden observar muchos rasgos concluyentes, como son los resultados académicos, características del contexto específico, rasgos relacionados con la 
personalidad o el estrato económico. En este sentido son determinantes las investigaciones recopilatorias, como el caso de Leyva (2017) que concluye que los estudios de género y educación deben completarse con un análisis interseccional de sexo, y su interacción con otras variables como la etnia o clase social (KOOMLER, 2017). Así en nuestra investigación se hace una descripción precisa de un estudio de caso, conscientes de sus limitaciones en tamaño, pero enriquecedor en aportaciones al fenómeno.

- Las conclusiones pueden diferir y no ir sistemáticamente en el mismo sentido. Así parece que el género no es determinante en ciertos componentes, como la autoeficacia de la actividad, las habilidades sociales y la inteligencia emocional (SALAVERA; USAN; JARIE, 2017). Y mientras ciertos registros descubren mejores resultados de los chicos en materias concretas (COBB-CLARK; MOSCHION, 2017; VALLE et al., 2016), otros trabajos llegan a la conclusión opuesta (YARBROUGH; CANNON; BERGMAN, 2017). Así, en nuestra investigación los efectos de la vida como estudiante de enseñanzas medias reducen las diferencias académicas entre alumnos y alumnas, mientras que en Rosales Mendoza y Salinas Quiroz (2017) se concluye de forma opuesta en que la enseñanza transmite estereotipos y prejuicios de género y sexualidad.

- La diferencia de casos entre los diversos países es acusada. Como se ha indicado este estudio analiza un caso específico en España como país intermedio, siendo conscientes de la disparidad de situaciones en determinados países con coyunturas de acusada marginación por razones de género y otros países, como los escandinavos, con entornos casi opuestos, (KAUFMAN; BERNHARDT; GOLDSCHEIDER, 2017).

- Es necesario extraer conclusiones concretas, más allá de la cuestión global de género, que impulsen y mejoren la práctica educativa. Esto es, son relativamente reducidos los trabajos que hacen propuestas precisas, tales como diseños metodológicos para el aula en los que se utilizan los resultados de la investigación. A modo de ejemplo podemos citar a Reychav, McHaney y Burke (2017) que infieren en cómo las alumnas usan estrategias de aprendizaje que pueden beneficiar el proceso de enseñanza dentro de una metodología grupal, dado que son más propensas a utilizar estrategias tales como escuchar activamente, hacer preguntas y solicitar información.

\section{Discusión y conclusiones}

En los casos y nivel educativo analizados, la investigación confirma el hecho de que las alumnas obtienen mejores resultados que los alumnos. Pudiera considerarse el hecho diferencial de la edad biológica, dado que las chicas se convierten en adultas antes que los chicos (TEMBOURY, 2009). Sería por tanto interesante comparar estos datos considerando el desarrollo psico-biológico del adolescente. Pero el dato nuevo y revelador indica que el paso por el centro educativo reduce esta diferencia de resultados. Así se observa que en cinco de las ocho materias analizadas disminuye significativamente la desigualdad entre calificaciones de ambos sexos, siendo la reducción total de esa divergencia de 0,65 puntos entre evaluaciones consecutivas. Esta variable sugiere que la acción formativa y su capacidad socializante e igualitaria contribuyen a paliar las diferencias y el sesgo de género en la mayoría de las materias salvo en la Lengua y Literatura, donde la actividad del docente iguala el rendimiento entre chicos y chicas 0,56 puntos como media. 
$\mathrm{Al}$ contrastar los resultados separados por materia observamos que las asignaturas donde los chicos están más cerca de las chicas son Tecnología y Educación Física. Patrón que se repite en ambas evaluaciones. Estas dos asignaturas pertenecen a la lista de asignaturas que tradicionalmente se han considerado de chicos. En cambio, Matemáticas aparece como una de las asignaturas en las que claramente las alumnas son más superiores a los chicos, en oposición a los resultados de Valle y otros autores (2016) y de Flecha (2014). También se observa como las asignaturas donde los estudiantes han sacado peores resultados son Matemáticas o Lengua, en coherencia con los hallazgos de González (2003). En cambio, materias en las que casi todos alcanzan un nivel suficiente, como de Tecnología y Educación Física, presentan una menor diferencia de nota media entre géneros. Esto sugiere la idea de que no existen asignaturas de chicos ni asignaturas de chicas y que al menos en este rango de edad las chicas obtienen mejores resultados sea cual sea la asignatura. Todo ello es un ámbito de relación interesante con las investigaciones de Salmi, Thuneberg y Vainikainen (2016) que correlacionan negativamente la actitud de hacia la ingeniería con los planes del futuro educativo que eligen los estudiantes de educación secundaria.

$\mathrm{Al}$ analizar los factores relacionados con los perfiles de altas, entre los estudiantes que destacan positivamente en las diferentes asignaturas aparecen en su mayoría chicas y es difícil encontrar chicos. En cambio, entre los estudiantes con peores resultados sí que se encuentran tanto chicas como chicos. Se podría afirmar que los mejores resultados estén en chicas, pero los peores estudiantes se reparten en ambas categorías y esto ocurre en todas las asignaturas de forma similar. Este hallazgo coincide con Goldschmidt y Bogner (2016), que también resaltan que las alumnas tienen unos mayores aumentos de nivel de conocimientos. Por ejemplo, la participación activa en las actividades prácticas de laboratorio resulta especialmente beneficioso para las mujeres, lo que supone una mayor eficiencia en las enseñanzas técnicas.

Sulaiman y AlMuftah (2010) destacan que posiblemente esté presente el influjo inicial de unas materias que han sido diseñadas desde una perspectiva masculina y para un desempeño masculino. Las razones de la diferencia de rendimientos pudieran deberse a la dificultad de la asignatura, dado que las materias donde la mayoría de estudiantes aprueban presentan mayor igualdad en los resultados, mientras que otras en las que hay muchas calificaciones bajas, la brecha es mayor. En este rango de edades, entre las chicas, se encuentran alumnas con muy buenas notas, pero también alumnas con malos resultados. En cambio, es difícil encontrar chicos brillantes, y la mayoría de ellos obtienen resultados normales o bajos. En el caso de la asignatura Tecnología, se observa que los chicos se acercan más a las chicas en rendimiento académico. Así, es la segunda asignatura en la que más se acercan las calificaciones medias entre hombres y mujeres, algo que apoyaría la teoría de que esta materia es más afín a los chicos. Parece que a medida que trascurre el curso los varones alcanzan en mayor medida el nivel de las chicas y éstas progresan, comparativamente, algo más lentamente en Tecnología.

Los resultados dan respuesta a los 4 objetivos iniciales en el sentido de:

a) Mayor rendimiento de las chicas en todas las asignaturas. 
b) Hay variaciones, supuestamente fruto de la acción educativa, incrementando el rendimiento académico en la asignatura de Lengua en las chicas, y disminuyendo esta supremacía en Música y Ciencias naturales.

c) Las variaciones por género en asignatura Tecnología se reducen con la acción educativa.

d) La exploración de la literatura científica confirma el subobjetivo a y b, no existiendo investigaciones sobre el subobjetivo c.

Al correlacionar los resultados de esta investigación con el análisis de literatura científica indexada y relacionada observamos la necesidad de matizar la tipología de cada caso analizado, por ejemplo, la diferencia de casos entre los diversos países es muy acusada. Es necesario hacer análisis desde una perspectiva objetiva, ya que se observa que las conclusiones pueden diferir y no ir sistemáticamente en el mismo sentido. No obstante, sería preciso aumentar el número de aportaciones y conclusiones concretas para su aplicación en el aula.

Estas referencias proporcionan argumentos sobre el nivel inicial del que parten los futuros alumnos y alumnas de enseñanza superior. Son datos que apuntan a una equiparación de perfiles como efecto de la acción académica. No obstante, coincidimos con Dobson (2012) al resaltar que estos crecimientos surgen de una base tan pequeña que es improbable que las mujeres se configuren como elemento dominante en las carreras técnicas y las ingenierías.

\section{Referencias}

ÁLVAREZ LIRES, María et al. La educación tecnocientífica: identificación masculina versus desidentificación femenina. Enseñanza de las Ciencias, Valencia, n. extra, p. 113-117, 2013. CONGRÉS D'INVESTIGACIÓ EN DIDÀCTICA DE LES CIĖNCIES, 9., 2013, Girona.

AVILA, Hector. Introducción a la metodología de la investigación. México, DC: Instituto Tecnológico de Cuauhtémoc, 1999.

BANDURA, Albert. Social foundations of thought and action. New Jersey: Prentice-Hall, 1986.

BEDDOES, Kacey; BORREGO, Maura. Feminist theory in three engineering education journals: 1995-2008. Journal of Engineering Education, New York, v. 100, n. 2, p. 281-303, 2011. Disponible en: <D0l: 10.1002/ j.2168-9830.2011.tb00014.x>. Acceso en: 2 my. 2013.

BORGONOVI, Francesca. ¿A qué tipo de carreras aspiran los chicos y las chicas? Pisa in Focus, París, v. 10, n. 3, p. 1-4, 2012.

CALANDRIA, Enma. Campañas en televisión contra la violencia de género del Ministerio de Sanidad, Política Social e Igualdad (2008-2011). Vivat Academia, Madrid, v. 15, n. 121, p. 17-30, 2012.

CEREZO, María Teresa; CASANOVA, Pedro. Diferencias de género en la motivación académica de los alumnos de Educación Secundaria Obligatoria. Revista Electrónica de Investigación Psicoeducativa, Madrid, v. 2 , n. 1, p. 97-112, 2004. 
CHOU, Pao Nan. Women studies in engineering education: content analysis in three referred journals. American Journal of Engineering Education, Taiwan, v. 4, n. 2, p. 99-104, 2013.

COBB-CLARK, Deborah; MOSCHION, Julie. Gender gaps in early educational achievement. Journal of Population Economics, Bonn, v. 30, n. 4, p. 1093-1134, 2017. Disponible en: <D0l: 10.1007/s00148017-0638-z>. Acceso en: 3 jul. 2017.

CUBILLAS, María et al. Gender stereotypes of college students in northern Mexico. Diversitas, Coahuila, v. 12, n. 2, p. 217-230, 2016.

DELGADO Beatriz et al. Diferencias de género y curso en metas académicas en alumnos de educación secundaria obligatoria. Revista Española de Pedagogía, Madrid, v. 68, n. 214, p. 67-84, 2010.

DOBSON, lan. It's a man's world: the academic staff gender disparity in engineering in $21^{\text {st }}$ Century Australia. Global Journal of Engineering Education, Ballarat, v. 14, n. 3, p. 213-218, 2012.

ECHAVARRI, Maximiliano; GODOY, Juan; OLAZ, Fabián. Gender differences in cognitive skills and academic performance in college students. Universitas Psychologica, Bogotá, v. 6, n. 2, p. 319-329, 2007.

EDEL NAVARRO, Rubén El rendimiento académico, concepto investigación y desarrollo. REICE, Bilbao, v. 1, n. 2, p. 1-15, 2003.

FLECHA, Consuelo. Desequilibrios de género en educación en la España Contemporánea: causas, indicadores y consecuencias. Areas, Sevilla, n. 33, p. 49-60, 2014.

FOMBONA, Javier. Lectura de imágenes y contenidos: competencias para el análisis de la forma y contenidos del audiovisual: hacia una teoría de la composición. Madrid: CEP, 2008.

FRADE, Alejandro. University students to the social networks: issues and user group in elitist structure or plural. Vivat Academia, Madrid, v. 13, n. 113, p. 1-13, 2010.

GOLDSCHMIDT, Marlen; BOGNER, Franz. Learning about genetic engineering in an outreach laboratory: influence of motivation and gender on students' cognitive achievement. International Journal of Science Education, v. 6, n. 2, p. 166-187, 2016. Part B - Communication and Public Engagement. Disponible en: <DOI: 10.1080/21548455.2015.1031293>. Acceso en: 2 en. 2017.

GONZÁLEZ, Rosa. Diferencias de género en el desempeño matemático de estudiantes de secundaria. Educación Matemática, México, DC, v. 15, n. 2, p. 129-162, 2003.

GUANIPA, María; MOGOLLÓN, Eddy. Estilos de aprendizaje y estrategias cognitivas en estudiantes de ingeniería. Revista Ciencias de la Educación, Carbobo, v. 1, n. 27, p. 11-28, 2006.

HAYAMIZU, Toshihiko; WEINER, Bernard. Cuestionario de educación de metas académicas. Journal of Experimental Education, London, v. 59, n. 3, p. 226-234, 1991.

KAUFMAN, Gayle; BERNHARDT, Eva; GOLDSCHEIDER, Frances. Enduring egalitarianism? Family transitions and attitudes toward gender equality in Sweden. Journal of Family Issues, California, v. 38, n. 13, p. 18781898, 2017. Disponible en: <DOI: 10.1177/0192513X16632266>. Acceso en: 3 my. 2017. 
KOOMLER, Alexis. A girl's education: schooling and the formation of gender, identities, and future visions. Journal of Youth and Adolescence, Berna, v. 46, n. 9, p. 2073-2077, 2017. Disponible en: <D0I: 10.1007/ s10964-017-0709-1>. Acceso en: 22 mzo. 2017.

LEYVA, Luis A. Unpacking the male superiority myth and masculinization of mathematics at the intersections: a review of research on gender in mathematics education. Journal for Research in Mathematics Education, New York, v. 48, n. 4, p. 397-433, 2017. Disponible en: <DOl: 10.5951/jresematheduc.48.4.0397>. Acceso en: 16 jun. 2017.

LLANERAS, Kiko. Género, educación y la brecha de las carreras técnicas. [S. I.: s. n.], 2015. Disponible en: <http:// politikon.es/2015/02/17/genero-educacion-y-la-brecha-de-las-carreras-tecnicas>. Acceso en: 21 my. 2016.

LYNN, Richard. Sex differences in intelligence and brain size: a paradox resolved. Personality and Individual Differences, v. 17, n. 2, p. 257-271, 1994. Disponible en: <D01: 10.1016/0191-8869(94)90030-2>. Acceso en: 16 jul. 2016.

MÍAS, Carlos. Habilidades verbales y no verbales para el aprendizaje y elecciones vocacionales para el ingreso universitario. Revista de Psicología, Córdoba, v. 9, n. 10, p. 156-76, 1999.

NAVARRO, Luis. ¿Para qué sirve la semiótica?: una propuesta de resignificación de la mujer a través de la comunicación para el cambio social. Investigación y Desarrollo, Barranquilla, v. 19, n. 1, p. 166-195, 2011.

ORTIZ, Adolfo; CANTO, Pedro. Estilos de aprendizaje y rendimiento académico en estudiantes de ingeniería en México. Journal of Learning Styles, México, DC, v. 6, n. 11, p. 160-177, 2013.

PARD0, Patricia; CALVET, María; MARTINEZ, Mari Carmen. Pioneer women in engineering studies: what can we learn from their experiences? European Journal of Engineering Education, v. 41, n. 6, p. 678-695, 2016. Disponible en: <DOI: 10.1080/03043797.2015.1121464>. Acceso en: 01 jul. 2016.

PAWLEY, Alice; SCHIMPF, Corey; NELSON, Lindsey. Gender in Engineering Education Research: A Content Analysis of Research in JEE, 1998-2012. Journal of Engineering Education, New York, v. 105, n. 3, p. 508528, 2016. Disponible en: <D0I: 10.1002/jee.20128>. Acceso en: 28 sept. 2016.

PERALES BLANCO, Verónica; GARCÍA GUARDIA, M. Luisa. Mujeres y tecnología. Icono 14, Madrid, v. 9, n. 1, p. 01-02, 2011.

REYCHAV, Iris; MCHANEY, Roger; BURKE, Daniel D. The relationship between gender and mobile technology use in collaborative learning settings: an empirical investigation. Computers \& Education, v. 113, p. 61-74, 2017. Disponible en: <DOI: 10.1016/j.compedu.2017.05.005>. Acceso en: 14 sept. 2017.

ROBINSON, David. Sex differences in brain activity, personality and intelligence: a test of arousability theory. Personality and Individual Differences, v. 25, n. 6, p. 113-115, 1998. Disponible en: <D0l: 10.1016/ S0191-8869(98)00113-5>. Acceso en: 30 jul. 2012.

ROSALES-MENDOZA, Adriana; SALINAS-QUIROZ, Fernando. Sex education and gender in Mexican elementary schools: what do textbooks and teachers have to say? Educare, Costa Rica, v. 21, n. 2, p. 223243, 2017. Disponible en: <D0I: 10.15359/ree.21-2.11>. Acceso en: 26 jun. 2017. 
SABANES, Dafne. Género y tecnología: capacitación para el activismo de las mujeres. Icono 14, Madrid, v. 9, n. 1, p. 101-128, 2011.

SALAVERA, Carlos; USAN, Pablo; JARIE, Laurane. Emotional intelligence and social skills on self-efficacy in secondary education students: are there gender differences? Journal of Adolescence, v. 60, p. 39-46, 2017. Disponible en: <D0I:10.1016/j.adolescence.2017.07.009>. Acceso en: 23 sept. 2017.

SALMI, Hannu; THUNEBERG, Helena; VAINIKAINEN, Mari. How do engineering attitudes vary by gender and motivation? Attractiveness of outreach science exhibitions in four countries. European Journal of Engineering Education, London, v. 41, n. 6, p. 638-659, 2016.

SULAIMAN, Noor; ALMUFTAH, Hend. A Qatari perspective on women in the engineering pipeline: an exploratory study. European Journal of Engineering Education, London, v. 35, n. 5, p. 507-517, 2010.

TEMBOURY, Mari Carmen. Desarrollo puberal normal: pubertad precoz. Revista Pediatría Atención Primaria, Madrid, v. 11, supl. 16, p. 127-142, 2009.

VALLE, Antonio et al. Actitudes hacia las matemáticas en estudiantes de educación primaria: diferencias en función del curso y del género, European Journal of Investigation in Health, Psychology and Education, v. 6, n. 2, p. 119-132, 2016. Disponible en: <D01: 10.30552/ejihpe.v6i2.161 >. Acceso en: 2 sept. 2016.

VÁZQUEZ, Stella. Rendimiento académico y patrones de aprendizaje en estudiantes de ingeniería. Ingeniería y Universidad, Bogotá, v. 13, n. 1, p. 105-136, 2009.

YARBROUGH, Jaime; CANNON, Laura, BERGMAN, Shawn. Let the data speak: gender differences in math curriculum-based measurement. Journal of Psychoeducational Assessment, London, v. 35, n. 6, p. 568580, 2017. Disponible en: <DOl: 10.1177/0734282916649122>. Acceso en: 3 febr. 2017.

Recibido en: 21.10 .2017

Revisiones en: 16.02.2018 Aprobado en: 24.04 .2018

Javier Fombona Cadavieco. Facultad de Formación de Profesores y Educación de la Universidad de Oviedo (España). Doctor en Ciencias de la Información y licenciado en Filosofía y Educación. Su trabajo trata la incorporación de las TIC en Educación. Ex director de TV, profesor desde 1986 en diferentes niveles. Actualmente coordina varios proyectos de investigación con dispositivos móviles y realidad aumentada.

Fernando Aragón Gutiérrez. Facultad de Formación de Profesores y Educación de la Universidad de Oviedo (España). Ingeniero industrial del departamento de electricidad y electrónica EDP España y Thyssenkrupp. Es controlador de instrumentación y organiza equipos avanzados.

María Ángeles Pascual Sevillano. Facultad de Formación de Profesores y Educación de la Universidad de Oviedo (España). Área de Didáctica y Organización Escolar. Catedrática de Escuela Universitaria. Investigadora en TIC y educación inclusiva. 